\title{
Truncated ARQ Based Cooperative Transmission Initialization
}

Faisal Raisham Khan, Dr. Muhammad Khalil Shahid

Institute of Communication Technologies

faisalrka@gmail.com

Institute of Communication Technologies

Khalildona@gmail.com

\begin{abstract}
A communication is energy efficient when the communicating peers are near to each other or where the separating distances are short and the LOS component is a dominant one. The chanaznel conditions in wireless communication vary with respect to time and distance. Let us consider a scenario where a terminal $X$ is communicating to another terminal $Y$ in a shadowed fading environment through an access point (AP) or a base station (BS). Supposing that the terminal $X$ is at the border of the transmission range of BS, any slight movement of the terminal X may result in either degradation of the wireless channel between terminal $X$ and BS or totally getting out of transmission radius of BS. The MAC layer of BS fails to receive the error-free packet as a result. The BS does not send back the ACK packet to the source terminal X. After waiting for a time specified, the source starts retransmitting the packet. If the source terminal does not receive the $A C K$ packet even after a threshold number of retransmissions $\Upsilon_{\text {max }}$ (RetryLimit), the source discards the packet. An efficient and reliable scheme of retransmission is proposed in this paper by using STBC based relay transmission system. MATLAB is used for simulation purpose and the results generated for direct and cooperative transmissions are compared in terms of the link layer parameter PER.
\end{abstract}

\section{Indexing terms/Keywords}

Cooperative Diversity, Packet Error Rate (PER), Maximum Retry Limit

\section{Academic Discipline And Sub-Disciplines}

Engineering (Telecom)

\section{SUBJECT CLASSIFICATION}

Wireless Communication

\section{TYPE (METHOD/APPROACH)}

Simulation

\section{Council for Innovative Research}

Peer Review Research Publishing System

Journal: INTERNATIONAL JOURNAL OF COMPUTERS \& TECHNOLOGY

Vol 13, No. 2

editor@cirworld.com

www.cirworld.com, www.ijctonline.com 


\section{INTRODUCTION}

Cooperative communications have gained much interest in the last decade due to its ability to mitigate the fading effect in wireless networks through spatial diversity without using multiple antennas on handheld and small devices. In cooperative communications, the source terminal transmits data to the destination terminal with the help of relay nodes. Generally, cooperative relaying systems have a source node that multicasts a data to a number of cooperating relay nodes, which resend a processed version of the received data to the intended destination node. The destination node combines the processed signals received from the relay nodes, taking into account the source's original signal [1], [2].

Cooperative communication depends upon the relaying techniques. Most commonly used methods for fixed relaying are: Decode-and-Forward (DF) in which the relays decode the message before forwarding to the destination, and Amplify-andForward (AF) in which relays simply repeat or amplify the received signal without decoding [1]. It has been shown [3] that the DF technique is slightly better than the AF in error probability and mutual information analysis. Moreover, a source node should partner with the ones that are closer in order to fully achieve the diversity order [3]. In [4] feedback channe state information (CSI) is employed at the transmitter for the initialization of cooperative communications. Due to the overhead of CSI, it is difficult to achieve high spectral efficiency and this becomes more energy consuming mechanism in the energy constraint wireless networks, like in sensor networks. In [5], a node cooperative stop and wait (NCSW) scheme is proposed for wireless channels with correlated frame error profiles. The authors proved the supremacy of cooperative $A R Q$ scheme in terms of throughput, delay jitter, and the average delay. Guanding $Y u$ at el. [6] proposed a simple $A R Q$ based cooperative protocol in which the received packets are not combined at the destination terminal. Their work presents data link layer packet error rate (PER) performance and spatial diversity. Hybrid-ARQ-based intra-cluster geographic relaying (HARBINGER) [7] shows significant improvement in the energy-latency tradeoff but it utilize expensive GPS (Global Positioning System) on each of the nodes for the selection of cooperating relays. In all these researches, the performance analysis has employed hard decision for the starting of cooperative communication, i.e. the cooperative communication is initialized by the source and potential relays upon receiving the first NACK packet from the destination.

In this paper, an ARQ based cooperative communication scheme is proposed in which the source terminal exploits the Medium Access Control (MAC) layer retransmission limit for the initialization of cooperative transmission without any CSI feedback overhead and present the performance analysis with various number of retransmission tries with different number of relaying nodes. Whenever this maximum retransmission limit is exceeded a fixed number predefined, the cooperative transmission is initialized. Nodes that participate in the cooperative communication operate in a time division duplex (TDD) mode to avoid interference [1].

The following sections of the paper describe problem statement along with the system model for wireless networks under consideration, the performance analysis of the proposed retransmission scheme, MATLAB® simulation results and the conclusions.

\section{COOPERATIVE RELAYING AND SYSTEM MODEL}

It has been shown [8] that peer to peer communication is energy efficient in short distances and, in the situations where the LOS (Line of Sight) component of the received signal is dominant (channel having less number of scattered signal components). In wireless mobile communication systems, the channel conditions do not remain constant. Consider a situation where a user $A$ is communicating with another user B through an Access Point (AP) or a Base Station (BS) in a shadowed fading environment. Let suppose that the user $A$ is at the edge of transmission range of Access Point. Some slight movement of user A may results in either getting out of transmission radius of AP or may cause degradation in wireless channel link between user A and AP. Due to such degradations, the MAC layer of AP fails to receive the packet without errors. MAC layer does not send the ACK message to the source, and after a specified time, retransmission of the packet is started. After a threshold number of retransmissions (Maximum RetryLimit), the source discards the packet if no $A C K$ is received. In the scheme proposed in this paper, the source terminal goes for relay transmission upon running out of the maximum retry limit (MRL) before discarding the packet. In this way, the channel state information (CSI) from MAC layer is utilized by the Physical layer for the start of source cooperative transmission. The maximum retry limit (MRL) can be set shorter for the delay sensitive applications.

The relay cooperative communication system consists of a source, destination and total of $\mathrm{N}$ relays. We analyze DF space-time block code (STBC) based cooperative MIMO (Multiple-Input-Multiple-Output) communication system as shown in the Figure 1 


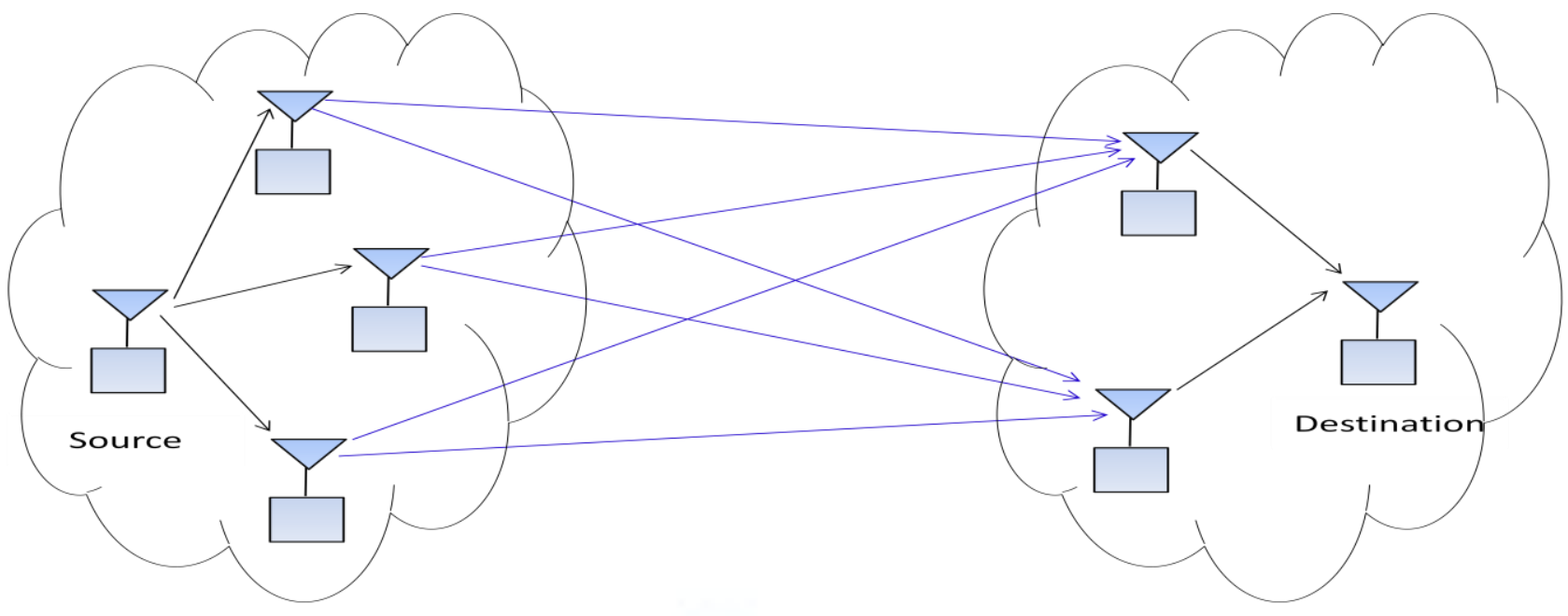

Trans-Cooperative Group

Receive-Cooperative Group

Figure 1: System Model

The transmission protocol follows three phases. In the first phase, the source detects the need for cooperative communication for reliable transmission to the destination. In the second phase, it multicasts the signals to the closest relay nodes for STBC based cooperative transmission. In third phase, the relay nodes send the processed version of the received signals to either the other relay nodes in the receive-cooperative group or directly to the destination. The nodes in receive-cooperative group, after receiving complete STBC block in third phase, send it to the destination through direct transmission. The channel is assumed to be quasi-static with flat fading i.e. the channel remains constant within one frame period, but it varies independently between the frames. We choose the Rayleigh channel model. An orthogonal STBC cooperative MIMO system with $m_{T}$ transmit and $m_{R}$ receive nodes is considered in this paper. Each orthogonal STBC is defined by an $m_{T} \times T_{B}$ transmission matrix [9]. Assuming that $n_{S}$ symbols are transmitted by the transmission matrix, the code rate $R_{C}$ will be defined as, $R_{C}=n_{S} / T_{B}$, where $T_{B}$ is the number of time periods for transmission of one block of coded symbols.

If each node in a transmitting cooperative group of $m_{T}$ nodes transmits a symbol with energy $\mathrm{E}_{s} / m_{T}$, the effective instantaneous SNR at the ML STBC decoder of the receiver is given by [10]

$$
\Upsilon_{s}=\frac{\|\mathbf{H}\|_{\mathrm{F}}^{2}}{m_{T} R_{c} N_{o}} E_{s}
$$

where $\|\mathbf{H}\|_{\mathrm{F}}^{2}=\sum_{i=1}^{m_{R}} \sum_{j=1}^{m_{T}}\left\|h_{i, j}\right\|^{2}$ is the squared Frobenius norm of $\mathbf{H}, h_{i, j}$ is the fading coefficient or channel gain from $j^{\text {th }}$ transmit antenna to the $i^{\text {th }}$ receive antenna.

If $P_{S}$ is the total transmission power, $P_{n}$ is the noise power then $S N R=P_{S} / P_{n}$. Choosing spectral efficiency equal to bandwidth, expression (1) can be written as

$$
\Upsilon_{s}=\frac{\|\mathbf{H}\|_{\mathrm{F}}^{2}}{m_{T} R_{c}} \cdot \frac{P_{s}}{P_{n}}
$$

For simplicity, the gains of the antenna are considered to be unity and we assume that there is no loss in the system hardware. As mentioned above, the channel fading is Rayleigh, i.e. the channel gain $h_{i, j}$ is a zero mean complex Gaussian random variable with independent real and imaginary components. The variance of the channel gain is $\sigma^{2}$. Depending upon the simplified path loss model, the channel strength $\sigma^{2}$ is given as

$$
\sigma^{2}=\left(\frac{\lambda}{4 \pi}\right)^{2} d^{-l}
$$

where $d$ is the average distance between the source group and destination group, $/$ is called path loss exponent and $\lambda$ is wavelength.

It is assumed that cooperating nodes area nearby the source node so that the large scale fading of relay-destination channels can be approximated to be the same as that of the source-destination channel. 
The total transmission power is distributed among the source and the selected relays and is given by

$$
P_{s}=\frac{S N R \cdot P_{n}}{\sigma^{2}}
$$

\section{PERFORMANCE ANALYSIS}

The instantaneous SEP for QPSK is given by [11]

$$
\psi=2 Q\left(\sqrt{\frac{E s}{N_{0}}}\right)-Q^{2}\left(\sqrt{\frac{E s}{N_{0}}}\right)
$$

As spectral efficiency is assumed to be equal to the bandwidth, the instantaneous SEP for QPSK in equation (5) can be re-written as

$$
\psi=2 Q \sqrt{S N R}-Q^{2} \sqrt{S N R}
$$

We assume Nyquist pulse shaping filter with bandwidth $\mathrm{B}$ and symbol period $T_{s}$ i.e. $T_{s} \approx 1 / B$. A packet is discarded if it is received erroneously after $\Upsilon_{\max }+1$ maximum number of transmissions i.e. after $\Upsilon_{\max }$ retransmissions. Therefore the packet loss probability at the link layer will not be greater than [12], [13]

$$
\left[1-(1-\psi)^{L / \log _{2} M}\right]^{\Upsilon_{\text {max }}+1}
$$

where $\psi$ is Symbol Error Rate or Symbol Error Probability at destination and $L$ is the packet length in bits.

The packet error probability when employing MRC over STBC in slowly varying block fading channel is given by combining the law of conditional probability and total probability as [14],

$$
P(\xi)=P\left(\xi \mid \Upsilon \geq \Upsilon_{\max }\right) P\left(\Upsilon \geq \Upsilon_{\max }\right)
$$

where $\Upsilon$ is the number of packet retransmission. The first term in expression (8) is packet loss probability, as the system discards the packet after $\Upsilon_{\max }$ number of retransmissions in order to avoid the lingering delays. The second term of the expression (8) is the probability of Channel State Information (CSI) without explicit feedback i.e. transmitter goes for cooperative transmission after the first NACK message is received from the receiver side. Combining the above expressions gives

$$
P(\xi)=\left[1-(1-\psi)^{L / \log _{2} M}\right]^{\Upsilon_{\max }+1}\left[1-(1-\psi)^{L / \log _{2} M}\right]
$$

\section{SIMULATION RESULTS}

In this section, computer simulations using MATLAB are provided. Performance is evaluated through link layer PER in Rayleigh fading environment. Results are compared with direct transmission with various maximum retry limit (MRL). Modulation scheme used is QPSK and carrier frequency is $2.5 \mathrm{GHz}$. Packet length is taken to be 20 bits. The average distance between the two cooperating groups is $100 \mathrm{~m}$. Direct transmission (DT) and various Cooperative transmissions (CT) are simulated using one million symbols for $0-30 \mathrm{db}$ SNR values with various MRL.

Fig. 2 depicts the system PER versus SNR for direct transmission. With varying MRL from 0 to 4 , it can be observed that the PER improves with MRL but it would take more time for a packet to be delivered successfully. 


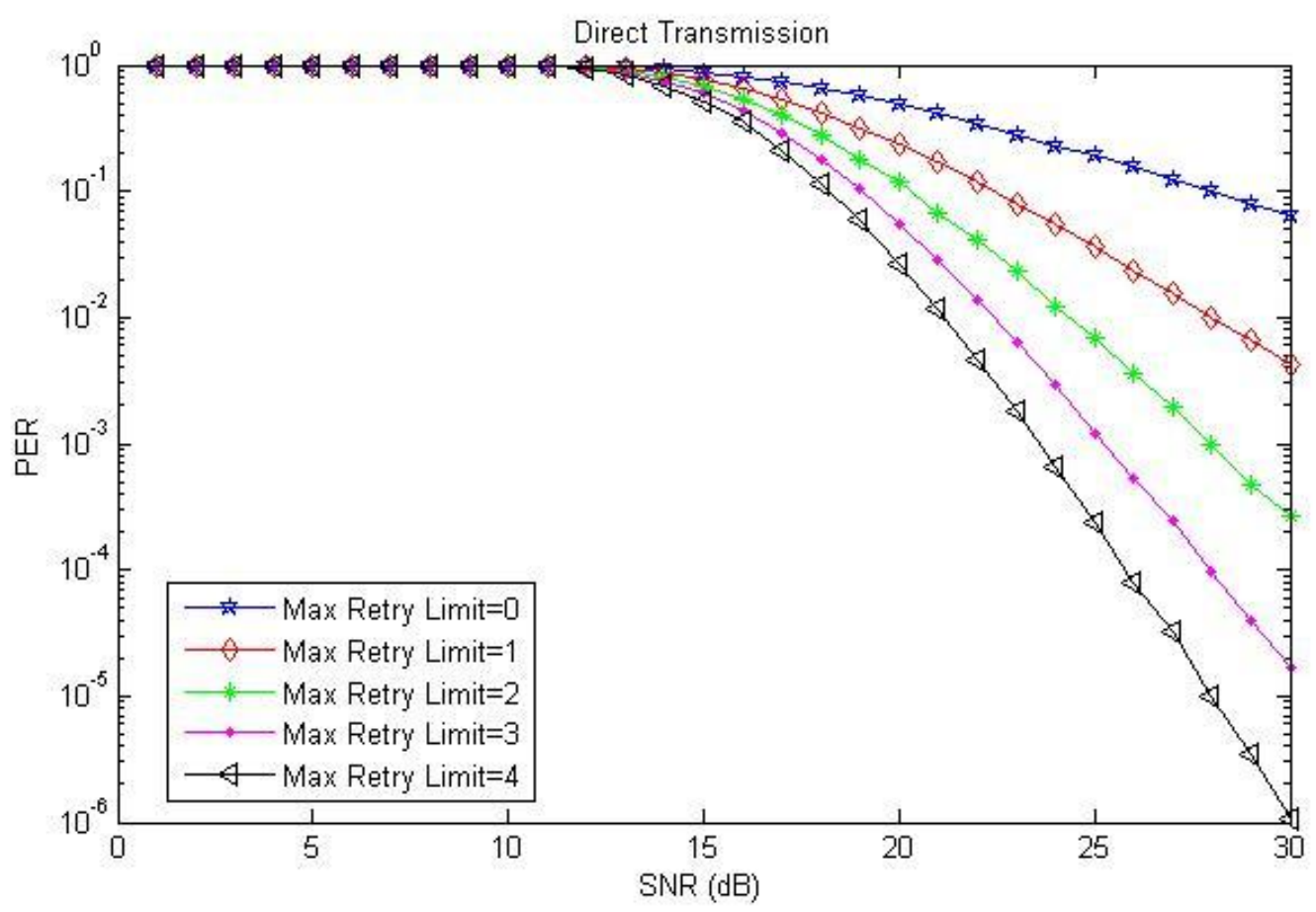

Figure 2: PER vs SNR for Direct Transmission

Fig. 3 shows the PER versus SNR for cooperative transmission system with 2 nodes as relays in Transmitting cooperative group and a single destination node in the receiving group. PER improves with MRL. It is clear from the fig. 2 and fig.3, that the PER performance is better for the cooperative transmission. Fig. 3 shows performance for a system with two fixed nodes as relays. Now a system with multiple nodes available as relays is considered. Two best relays are selected from $\mathrm{N}$ available relays on the basis of better BER performance for the links between the relays and destination. It can be shown that PER improves with increase in $\mathrm{N}$. It is because two best relays are selected among the $\mathrm{N}$ potential nodes. Fig.4 shows the performance of such system with $\mathrm{N}=6$ nodes.

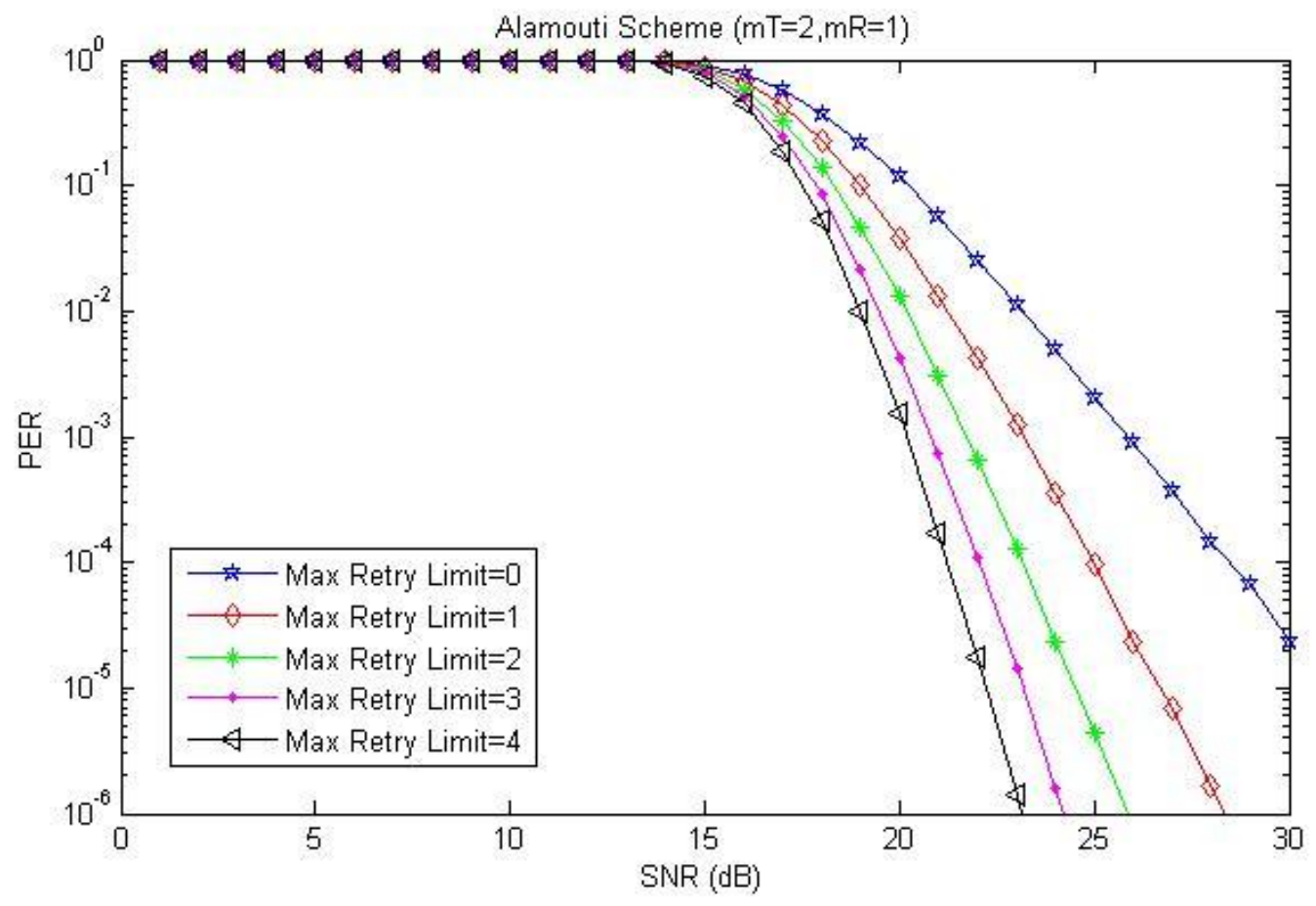

Figure 3: PER vs SNR for CT with two fixed relay nodes in Trans-Cooperative Group and a single Destination node in Receive-Cooperative Group 


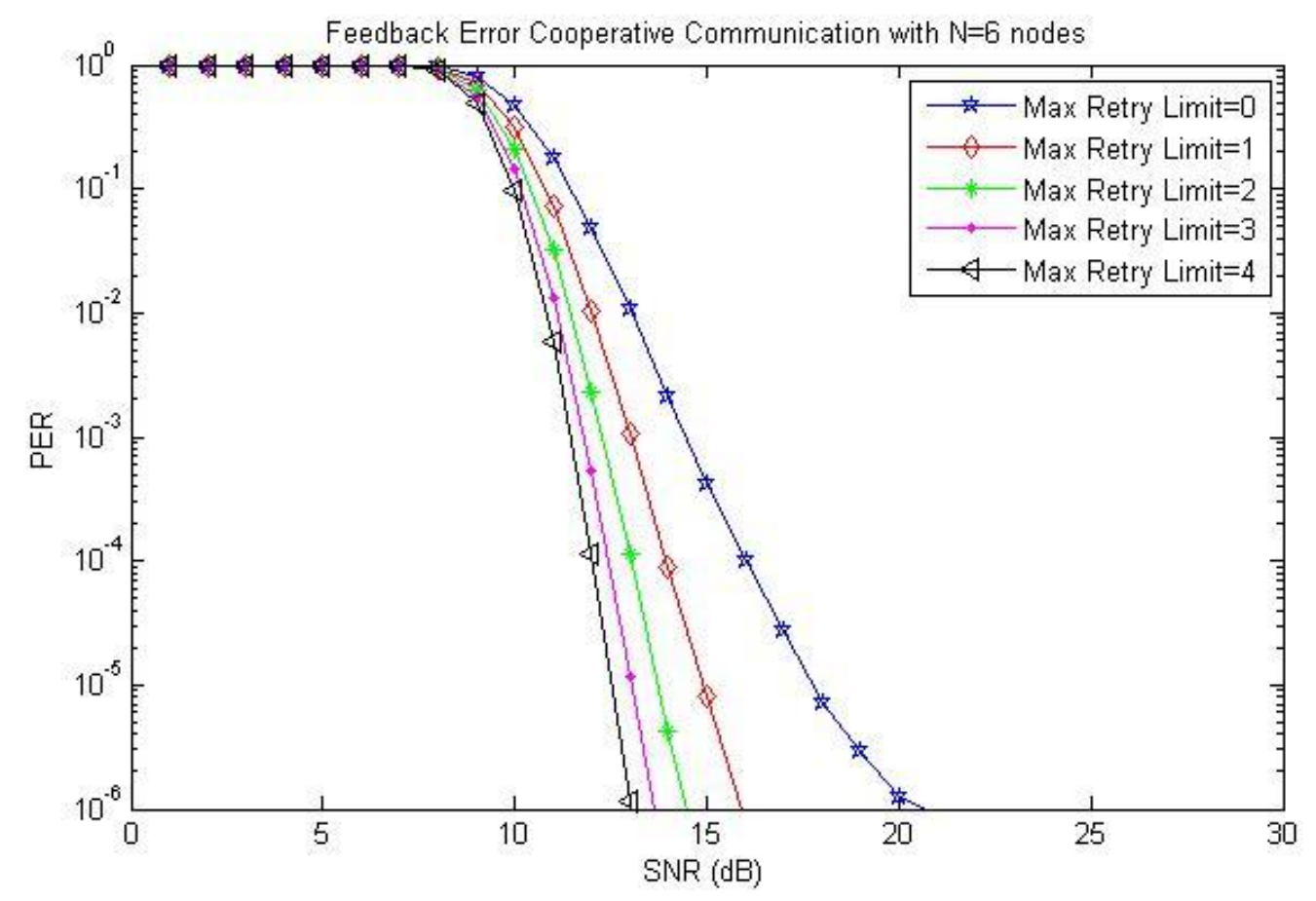

Figure 4: PER vs SNR for CT with selection of two best relay nodes from $\mathrm{N}=6$ available nodes in TransCooperative Group and a single Destination node in Receive-Cooperative Group

Cooperative transmission with three nodes as relays in source group and 2 nodes as relays in receiving group, creating a multihop transmission, is simulated for various values of $M R L$ and the results are given in Fig. 5 . Comparing the systems simulated in fig. 4 and fig. 5 , it can be observed that the system with more relays in source group and receive group is having better performance.

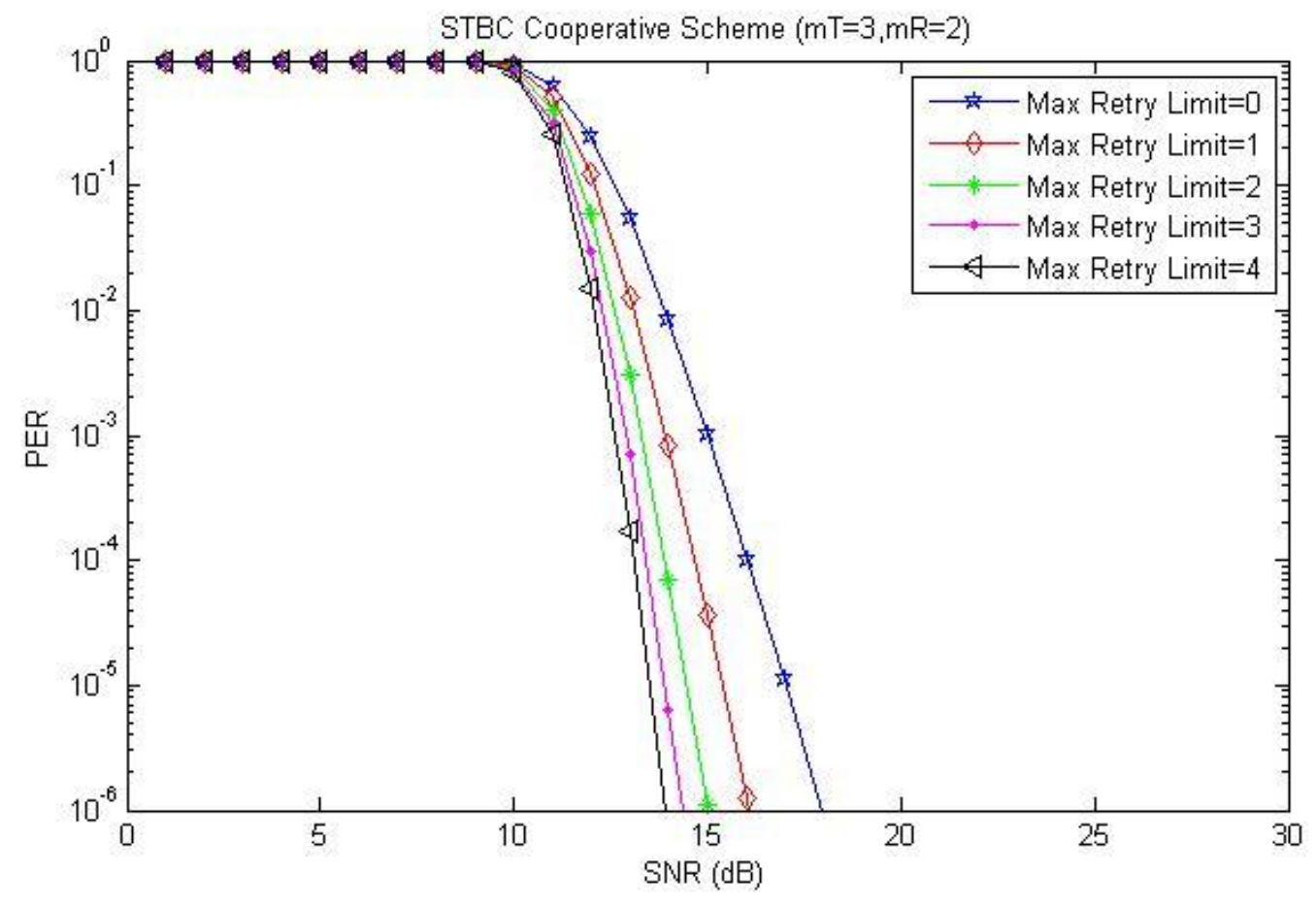

Figure 5: PER vs SNR for CT with three relay nodes in Trans-Cooperative Group and two relay nodes ReceiveCooperative Group 
Fig. 6 shows the comparison of systems with various transmission schemes having different MRL. The figure compares the cooperative systems with multiple nodes as relay in source group and only a destination node in the receiving group. It can be seen that the system with two fixed nodes as relays in source group and a single destination node in the destination group having MRL of only 1, outperforms the direct transmission with MRL value of 4 in high SNR regime. Other systems with no retransmissions are having much better PER performance as compared to the direct transmission with MRL value of 4 . It means that the STBC based cooperative transmissions achieve a better PER performance at link layer with a single transmission than what a direct transmission system achieves after a total of 5 transmissions.

Fig. 7 compares the direct transmission with MRL of 4 (i.e. total of 5 transmissions) and various cooperative transmission systems having multiple nodes as relays in trans-cooperating group as well as in receive-cooperating group with single transmission (i.e. no retransmission). All the cooperative transmission schemes are having much better performance with a single transmission than the direct transmission scheme with a total of 5 transmissions.

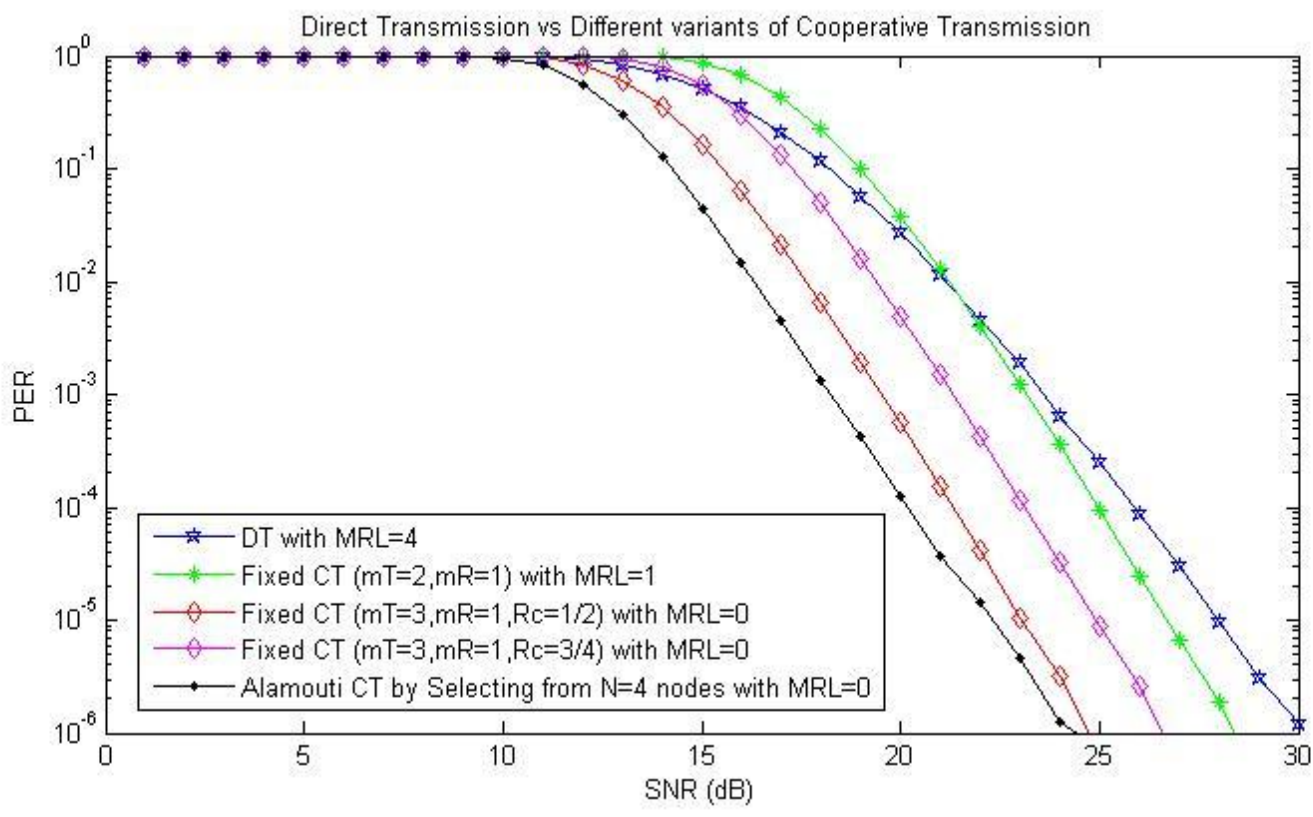

Figure 6: Comparison of DT and various CTs with different MRL

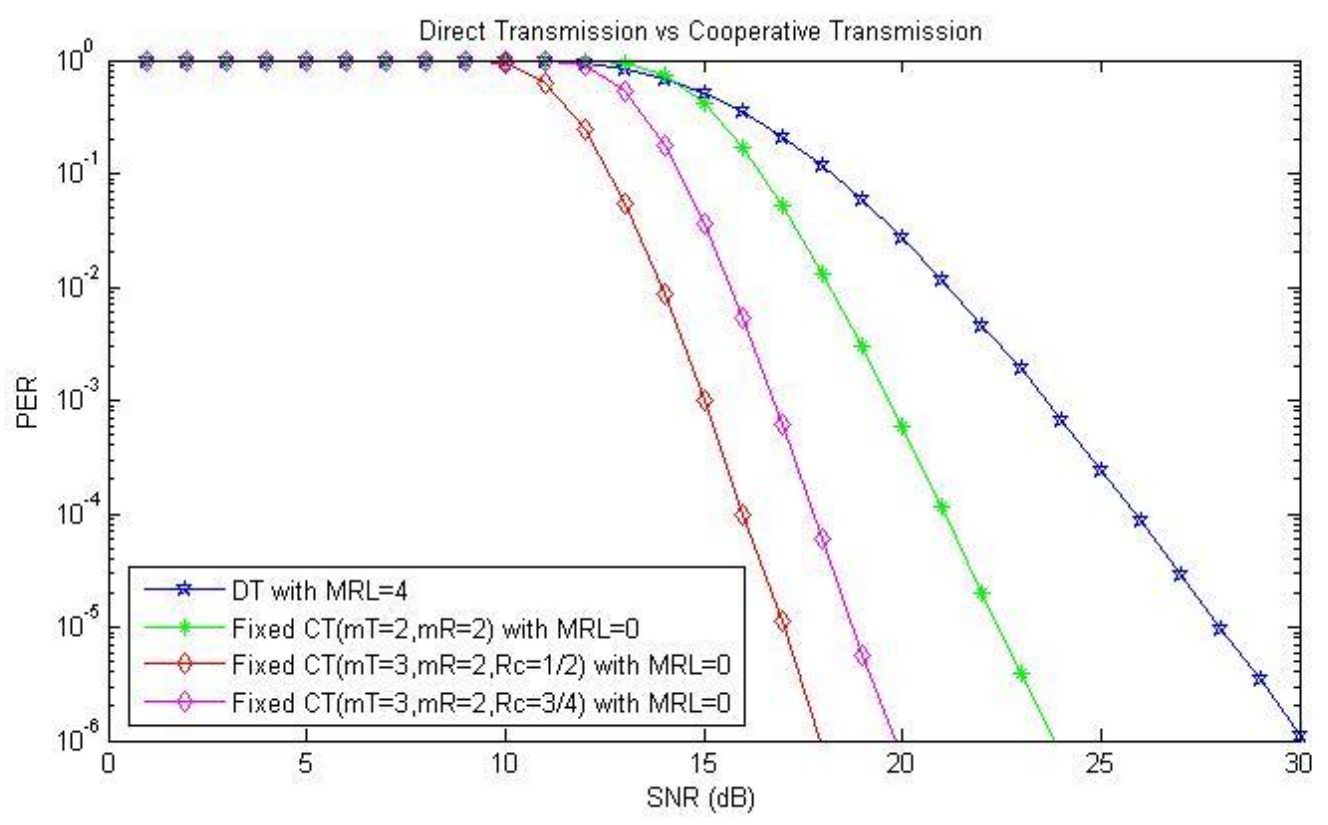

Figure 7: Comparison of DT and various CTs with different MRL 


\section{CONCLUSIONS}

In this work, various transmission schemes are developed and compared them with each other and with the direct transmission or non-cooperative transmission as reference. MATLAB simulations for each of them with different number of cooperating nodes have been carried out. All the transmission schemes are evaluated through Packet Error Rate with Max Retry Limit from 0 to 4 . It has been shown that the PER improves with MRL for direct transmission and all the other cooperative transmission schemes. The STBC based cooperative transmissions schemes achieved the same PER with no retransmission and 5-7 db gain in SNR, which would be achieved by direct transmission after a total of 5 transmissions. It can be concluded that the scheme of retransmission using STBC based relay transmission is much more efficient and reliable. Also, as the retransmissions are reduced, the scheme is having reduced delay.

\section{ACKNOWLEDGMENTS}

Our thanks to the experts who have contributed towards development of this paper.

\section{REFERENCES}

[1] J. N. Laneman, D. Tse, and G. W. Wornell, "Cooperative diversity in wireless networks: Efficient protocol and outage behavior," IEEE Trans. On Information theory, vol. 50, no. 12, pp. 3062--3080, Dec 2004.

[2] A. Bletsas, A. Khisti, D. P. Reed, and A. Lippman, "A simple Cooperative diversity method based on network path selection," IEEE J. Sel. Areas Commun., vol. 24, pp. 659-672, Mar. 2006.

[3] Meng Yu, Jing Li; "Is amplify-and-forward practically better than decode-and-forward or vice versa?" Acoustics, Speech, and Signal Processing, 2005 Proceedings. (ICASSP '05). IEEE International Conference on Volume 3, 18-23 March 2005 Page(s): iii/365 - iii/368

[4] Ahmed S. Ibrahim, Ahmed K. Sadek, W.Su, K.J. Ray Liu, "Relay Selection in Multi-node Cooperation Communications: When to Cooperate and Whom to Cooperate with?," IEEE Globecom 2006.

[5] Dianati, M.; Xinhua Ling; Naik, K.; Xuemin Shen, "A Node-Cooperative ARQ Scheme for Wireless Ad hoc Networks," IEEE Trans. On Veh Tech., Vol. 55, No. 3, May 2006.

[6] Guanding Yu, Zhaoyang Zhang, and Peiliang Qiu, "Cooperative ARQ in Wireless Networks: Protocols Description and Performance Analysis," IEEE ICC 2006.

[7] Bin Zhao, Matthew C. Valenti, "Practical Relay Networks: A Generalization of Hybrid-ARQ," IEEE J. on Select. Areas in Comm., Vol. 23, No. 1, January 2005.

[8] S. Cui, A. J. Goldsmith, and A. Bahai, "Energy-efficiency of MIMO andCooperative MIMO in Sensor Networks," IEEE J. Select. Areas of Communications, pp. 1089-1098, Vol. 22, No. 6, August, 2004.

[9] V. Tarokh, H. Jafarkhani, and A. Calderbank, "Space-time block codes from orthogonal designs," IEEE Transactions on Information Theory, vol. 45, no. 5, pp. 1456-1467, 1999.

[10] Hao Zhang Gulliver, T.A, "Capacity and error probability analysis for orthogonal space-time block codes over fading channels," IEEE Transactions on Wireless Communications, Volume: 4, Issue: 2 page(s): 808- 819, March 2005

[11] J. G. Proakis, Digital Communications, 4th ed. New York: McGraw-Hill, Inc., 2001

[12] Qingwen Liu, Shengli Zhou and Georgios B. Giannakis, Cross-Layer Combining of Adaptive Modulation and Coding With Truncated ARQ Over Wireless Links, IEEE TRANS. Wireless Commun., vol. 3, no. 5, 2004, pp. 1746-1755

[13] L. Dai and K. B. Letaief, "Cross-layer design for combining cooperative diversity with truncated ARQ in ad-hoc networks," Proc. IEEE Globecom, St. Louis, USA, pp. 3175-3179, Nov.-Dec. 2005.

[14] Irfan Ahmed, Mugen Peng, Wenbo Wang, Performance Analysis of an ARQ Initialized Cooperative Communication Protocol in Shadowed Nakagami-m Wireless Channel, IEEE Communications Society, ICC 2008 workshop proceedings, pp321-325, IEEE, 2008

\section{Author' biography with Photo}

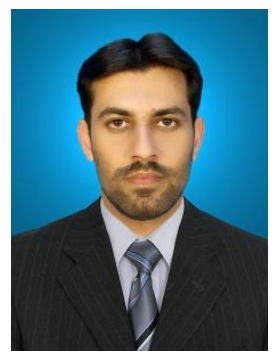

Faisal Raisham Khan: He has done his bachelor's degree in Electrical Engineering and persuing Master's degree in Telecommunication Engineering. He has worked in Pakistan Telecommunication Company Limited and currenty working in Telecom Department of Utility company. 